\title{
The Role of Multifocal Electroretinography in the Assessment of Drug-Induced Retinopathy: A Review of the Literature
}

\author{
Maria Dettoraki Marilita M. Moschos \\ Department of Ophthalmology, School of Medicine, National and Kapodistrian University of Athens, Athens, Greece
}

\section{Key Words}

Drug-induced retinopathy · Multifocal electroretinography · Retinal toxicity

\begin{abstract}
Multifocal electroretinography (mfERG) is an objective, noninvasive examination for the assessment of visual function. It enables the stimulation of multiple retinal areas simultaneously and recording of each response independently, providing a topographic measure of retinal electrophysiological activity in the central $40-50^{\circ}$ of the retina. A clinical application of mfERG represents the assessment of retinal toxicity associated with systemic medications. Drug-induced retinopathy represents a disease that, although not common, requires early recognition: if not detected early, it may progress and cause irreversible retinal dysfunction with subsequent visual impairment. This review aims to evaluate the use of mfERG in the assessment of retinal dysfunction associated with various systemic pharmacological agents based on the currently available literature. The most commonly recognized systemic medications affecting retinal function are included, such as chloroquine and hydroxychloroquine, vigabatrin, deferoxamine, ethambutol, interferon- $a$, tamoxifen, digoxin, sildenafil, canthaxanthin, amiodarone and nefazodone. The role of $\mathrm{mFERG}$ in the early diagnosis of retinal
\end{abstract}

toxicity and the evaluation of disease severity is reviewed, as well as its clinical value in monitoring disease progression or recovery after drug cessation.

(c) 2016 S. Karger AG, Basel

\section{Introduction}

\section{Multifocal Electroretinography}

Multifocal electroretinography (mfERG) represents an objective, noninvasive examination for the assessment of visual function. Since its first description by Sutter and Tran [1] in 1992, mfERG has dramatically changed the objective evaluation of retinal diseases. It enables stimulation of multiple retinal areas simultaneously and recording of each response independently, providing a topographic measure of retinal electrophysiological activity. Under light-adapting conditions and through dilated pupils, many local retinal responses, typically 61 or 103, are recorded, which represent the central and mid-peripheral visual field (VF), allowing the detection of small areas of retinal dysfunction in this region [2]. The retina is stimulated with an array of hexagonal elements, and each hexagon is alternated between black and white according to a pseudorandom sequence, called binary m-sequence, at a rate of $75 \mathrm{~Hz}$. Electrical responses from the retina are

\section{KARGER}

E-Mail karger@karger.com

www.karger.com/ore
(C) 2016 S. Karger AG, Basel

0030-3747/16/0564-0169\$39.50/0
Assoc. Prof. Marilita M. Moschos, MD, $\mathrm{PhD}$

Department of Ophthalmology, School of Medicine

National and Kapodistrian University of Athens

6 Ikarias Street, Ekali, GR-14578 Athens (Greece)

E-Mail moschosmarilita@yahoo.fr 
recorded with a corneal electrode as in full-field ERG recording [3]. The resulting waveforms consist of an initial negative component (N1), followed by a positive component (P1) and a second negative response (N2). mfERG responses represent mainly the function of the outer retina, such as the photoreceptors and the on- and off-bipolar cells [4]. mfERG stimulus location and anatomical areas correspond roughly to the following five concentric rings: ring 1 to the fovea, ring 2 to the parafovea, ring 3 to the perifovea, ring 4 to the near periphery and ring 5 to the central part of the middle periphery. The mfERG can also provide a graphical display of the signal amplitudes in a three-dimensional 'hill of vision' with the largest signals corresponding to the fovea.

The mfERG waveform can be distorted in cases of poor fixation, unstable electrode contact, errors in refraction or continuous blinking, and it should be taken under consideration that smaller amplitudes occur in older individuals and high myopes [5].

mfERG is a useful tool in detecting abnormalities in the macular, perimacular and mid-peripheral retina not being obvious on fundus examination, such as in chloroquine (CQ) [6] or hydroxychloroquine (HCQ) [7] toxicity, siderosis [8], anorexia nervosa [9], tilted disk syndrome [10] and keratoconus [11]. mfERG can objectively detect the loss of central activity in all kinds of maculopathies, including age-related macular degeneration, central serous chorioretinopathy, vitelliform maculopathy, macular hole, juvenile retinoschisis and other diseases [12]. With mfERG, the extent of the central lesion can be estimated in early stages of Stargardt's maculopathy and toxic maculopathy [12]. Moreover, mfERG in conjunction with visual evoked potentials (VEPs) is valuable in the differential diagnosis of retinal and optic nerve diseases [13].

Wide-field (WF)-mfERG has the potential to stimulate more peripheral retinal areas in comparison with conventional mfERG. Approximately $90^{\circ}$ of the retina can be detected with the use of WF-mfERG, whereas the testing field of conventional $\mathrm{mfERG}$ is around $40-50^{\circ}$ [4]. It is used in assessing peripheral retinal dysfunction in patients with retinitis pigmentosa [14], retinal vein occlusion [15], birdshot chorioretinitis [16] and vigabatrin (VGB) toxicity [17, 18].

\section{Retinal Toxicity}

Drug-induced ocular toxicity consists of a diverse group of conditions in clinical ophthalmology that, although not common, requires early recognition. Most ocular side effects are reversible after cessation of therapy if detected early. However, if the toxic effects are not detected, they may progress and cause advanced and potentially irreversible ocular dysfunction with associated visual loss [19].

Ocular side effects occur not only from topical application of drugs but also from their systemic administration. There are a large number of drugs that can cause various degrees of ocular toxicity, involving the eyelids, cornea, lens, uvea, optic nerve and retina. We have included the most commonly recognized systemic medications affecting retinal function, including CQ and HCQ, VGB, deferoxamine, ethambutol, interferon- $\alpha$, tamoxifen, digoxin, sildenafil, canthaxanthin, amiodarone and nefazodone.

The assessment of retinal toxicity is based on a complete medical history and an ophthalmologic examination that includes visual acuity measurement, color vision tests and fundoscopy after pupil dilatation. However, beyond routine ocular examination, other investigations allow detailed evaluation of retinal structure and function, such as optical coherence tomography (OCT), perimetry, fundus autofluorescence (FAF), fundus angiography and visual electrophysiology, including mfERG.

This review aims to evaluate the use of mfERG in the assessment of retinal dysfunction associated with various systemic pharmacological agents based on the currently available literature. The role of $\mathrm{mfERG}$ in the early diagnosis of retinal toxicity and evaluation of disease severity is reviewed, as well as its value in the monitoring of disease progression or recovery after drug cessation.

In the following paragraphs, the findings of mfERG examinations in retinal toxicity induced by different medications will be reviewed.

\section{CQ and HCQ Retinopathy}

CQ and HCQ are antimalarial drugs with a well-established beneficial role in the treatment of rheumatoid arthritis, systemic lupus erythematosus and other connective tissue and skin disorders. CQ and HCQ are melanotropic drugs that become concentrated in melanincontaining structures of the eye, such as the iris, ciliary body, retinal pigment epithelium and choroid. The ocular side effects include keratopathy, ciliary body changes and retinopathy [20]. CQ and HCQ retinopathy is characterized by pigmentary changes that vary from subtle stippling to a granular pigmentation in the macula, surrounded by a depigmented zone of retinal pigment epithelium atrophy, which is encircled by a hyperpigmented 
ring. The described fundus appearance is referred to as 'bull's eye maculopathy'. Blurred distance vision, photophobia, central VF scotomas and difficulty in reading have been described in CQ and HCQ toxicity [20].

It is considered that the use of CQ provides a greater risk for retinopathy than HCQ, probably because CQ passes the blood-retinal barrier [21]. Although HCQ is usually less disruptive to the retina than CQ, mfERG changes can still occur.

mfERG has been widely used in CQ/HCQ retinopathy. In 1999, Maturi et al. [22] first performed mfERG in a HCQ-treated patient with pigment mottling in the macula in both eyes and reported a markedly decreased electrical response in the macula in both eyes. Subsequently, the other authors confirmed the value of mfERG in detecting early retinal dysfunction in CQ/HCQ toxicity before the development of fundus abnormalities or visual impairment [6, 23-30]. In a study by Moschos et al. [31], 4 of 11 patients under HCQ treatment had normal electrooculogram (EOG) responses but reduced mfERG responses, and 1 patient presented with subnormal EOG and normal mfERG; they concluded that mfERG may be a more sensitive method than EOG in detecting HCQ retinopathy. Missner and Kellner [32] compared different screening methods (mfERG, color vision test, perimetry, ophthalmoscopy and fluorescein angiography) in CQ/HCQ retinopathy and reported that the use of mfERG may detect retinal dysfunction in a large number of eyes with normal fundoscopy and fluorescein angiography. The color vision and VF testing results were characterized by a higher variability, which suggests the use of $\mathrm{MfERG}$ as primary screening tool for retinal toxicity in long-term CQ/HCQ treatment. A more recent study reported that the functional changes elicited by mfERG and VF examination precede the anatomical changes demonstrated by spectral-domain (SD)-OCT in patients screened for HCQ retinopathy [33]. The American Academy of Ophthalmology revised recommendations concerning mfERG for screening of CQ and HCQ retinopathy, published in 2011, including the possible replacement of VF examination with the objective mfERG examination [34]. It was also emphasized that the goal of screening is to recognize toxicity at an earlier stage. Browning and Lee [35] studied the relative sensitivity and specificity of mfERG compared with 10-2 VF and SD-OCT in 102 patients taking CQ and HCQ. The sensitivities of mfERG, 10-2 VF and SD-OCT in detecting CQ/HCQ retinopathy were 92.9, 85.7 and $78.6 \%$, respectively, and the specificities of mfERG, 10-2 VF and SD-OCT were 86.9, 92.5 and 98.1\%, respectively. mfERG demonstrates most commonly a paracentral ring

mfERG to Assess Drug-Induced Retinopathy depression of signals around the fovea [24, 25, 27, 28, 36]. Kellner et al. [6] compared mfERG responses to FAF findings and showed that only 8 of 13 patients under CQ/HCQ therapy with abnormal mfERG responses had FAF abnormalities. The pericentral amplitude was first affected with a progression to central amplitude reduction and generalized function loss, and finally with completely unmeasurable responses in advanced retinopathy. The degree of amplitude reduction was found to correspond to the severity of morphologic alterations in FAF imaging, though without a direct association between mfERG and FAF patterns. In order to evaluate the second-order adaptation effects in patients taking HCQ, Penrose et al. [37] used a special mfERG stimulus, which revealed early detection of HCQ retinopathy, but some patients, although having normal second-order responses to the new stimulus, still had abnormal first-order responses to the classic mfERG stimulus. The authors suggested that fast inner retinal feedback mechanisms may play a role in the discrepancies observed between first- and second-order effects. Maturi et al. [24] reported that the duration of HCQ therapy is an important factor in the appearance of retinopathy. They observed that 10 of 11 patients with abnormal mfERG responses had taken HCQ at the recommended dose for at least 5 years. So et al. [26] showed pericentral depression of mfERG signals in 3 of 6 patients treated with HCQ. All affected patients had been taking HCQ for at least 7 years. Recently, Leung et al. [36] found that high doses of HCQ can lead to retinal toxicity within 1-2 years. Widespread signal depression across the macula and especially in the parafoveal region was observed with mfERG in 2 of 7 patients studied. Therefore, it is suggested that patients who take HCQ at doses higher than the recommended dose ( $>6.5 \mathrm{mg} / \mathrm{kg}$ per day) or for a long duration ( $>5$ years) should be considered for periodic mfERG testing for the early detection of HCQ toxicity [24, 38]. Moschos et al. [39] demonstrated that mfERG amplitudes were markedly improved 6 months after HCQ cessation for both central and peripheral responses (fig. 1). The reversibility of HCQ toxic effects detected by mfERG has also been demonstrated by other authors [6, 24, 27, 29, 31]. This suggests that early toxicity is probably reversible, and the detection of CQ/HCQ retinopathy at the subclinical stage is necessary in order to prevent permanent toxicity.

mfERG is, therefore, considered an objective examination of retinal function in patients taking CQ or HCQ that can provide earlier and more reliable detection of retinal damage in these patients. mfERG is also useful in confirming the absence of retinal toxicity when perimetry or other tests detect abnormalities. 
Fig. 1. mfERG changes in a patient with HCQ retinopathy. a The three-dimensional topography of mfERG demonstrates a subnormal central region and the ERG traces of areas 1 and 2 are very low. b Six months after the interruption of the drug, there is an improvement in the three-dimensional appearance of $\mathrm{mfERG}$ and ERG traces [from ref. 31, with permission].

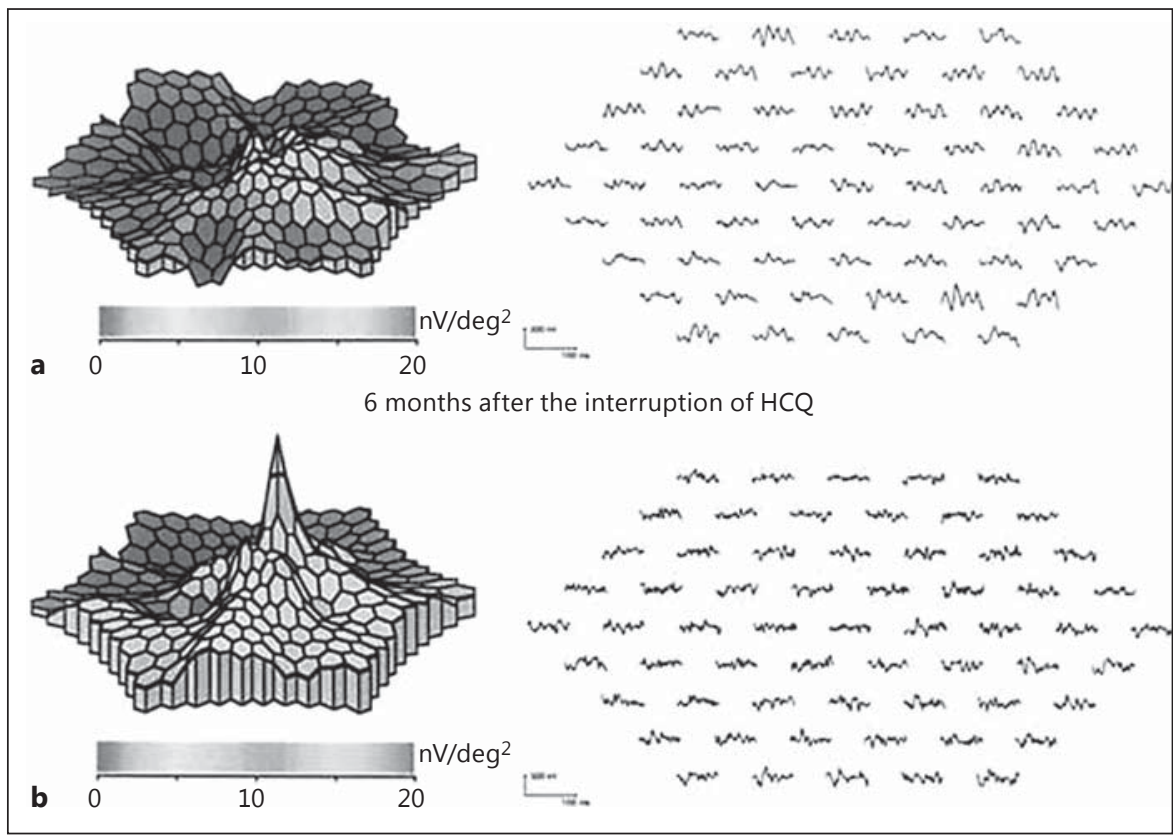

\section{VGB Retinopathy}

VGB is an irreversible inhibitor of the enzyme $\gamma$ aminobutyric acid transaminase used in the treatment of epilepsy. A common ocular complication of VGB toxicity is bilateral VF constrictions [40, 41].

mfERG has been commonly used to evaluate VBG retinopathy. Electrophysiological findings in VGB toxicity have confirmed that the deficit occurs at the retinal level [42-46]. It has been demonstrated that patients with VF defects associated with VGB had diffuse loss of function or reduction in amplitudes of peripheral focal ERG. In some patients, the abnormalities in MfERG amplitudes appeared to correspond well with VF defects $[43,47]$, whereas in other patients the abnormalities may be more diffuse than VF findings [43] or have a poor correlation $[42,45]$. Besch et al. [48] observed delayed multifocal oscillatory potentials in patients taking VGB with marked VF defects but without visual complaints and with normal visual acuity, color vision and dark adaptation thresholds. This finding indicates that VGB-related VF defects may be a result of inner retinal dysfunction. Gonzalez et al. [17] investigated visual defects associated with VGB with the use of static perimetry and WF-mfERG in 204 epileptic patients with and without exposure to VGB. Interestingly, bilateral VF defects were observed in almost half of the patients with
VGB use and in $24 \%$ of patients never exposed to the drug, but abnormal ERG was detected only in patients with exposure to VGB. The authors concluded that a proportion of patients with epilepsy have binasal field defects that cannot be attributed to VGB exposure, and perimetry alone cannot prove VGB-related retinal toxicity. They suggested that an objective measure of retinal function, such as WF-mfERG, should ideally be used to assess VGB-associated toxicity. Using WF-mfERG, McDonagh et al. [18] examined the retinal function of VGB-treated patients and reported that WF-mfERG can be expected to be a good predictor of field loss with $100 \%$ sensitivity and $86 \%$ specificity. Moreover, mfERG may become especially valuable in monitoring patients taking VGB who do not cooperate in VF testing, such as young children, in order to prevent VF defects. Studies have shown that VF defects persist when VGB therapy is withdrawn, which makes the early diagnosis of VGB toxicity imperative $[40,42,43]$.

mfERG findings are considered useful and more sensitive indicators of early retinal dysfunction than visual acuity, color vision, VF and dark adaptation in patients taking VGB. Moreover, mfERG is able to exclude retinal toxicity in patients with VF defects not associated with VGB intake. 


\section{Deferoxamine Retinopathy}

Deferoxamine mesylate (DFO) is the most used ironchelating drug for the treatment of hemosiderosis secondary to transfusions in patients with sickle cell anemia, $\beta$-thalassemia, myelodysplastic syndrome, aplastic anemia and myelofibrosis for example. DFO is most commonly administered as a slow subcutaneous infusion but can also be given intramuscularly or intravenously [49]. Ocular findings of DFO toxicity include cataract, optic neuropathy and retinopathy [50-52]. Retinal abnormalities include macular and peripheral pigmentary changes, retinal pigment epithelium degenerations and bull's eye maculopathy [53-55].

Using mfERG, Schmidt and Finke [56] showed reduced amplitudes (approximately half of the normal values) in the central retina of a patient who developed bull's eye maculopathy after DFO therapy. Kertes et al. [57] demonstrated bilateral reductions in response densities at the central retina that corresponded with the pigmentary changes developed due to DFO toxicity. Serial mfERG recordings allowed objective quantifiable monitoring of functional changes developing over time in DFO maculopathy. The authors observed progressive worsening of mfERG traces during the first 4 months after cessation of DFO therapy, which did not stabilize until 8 months following discontinuation of the drug.

Despite the small number of cases reported, mfERG seems a useful tool to diagnose DFO retinopathy, and to locate and assess the progression of retinal dysfunction.

\section{Ethambutol Retinopathy}

Ethambutol is used for the treatment of tuberculosis and Mycobacterium avium complex infections. Ocular toxicity associated with ethambutol includes optic neuropathy, VF defects and impaired color vision [58].

mfERG was performed in a patient with bilateral visual loss while on ethambutol therapy for the first time by Lai et al. [59] in 2004. Bilateral toxic maculopathy was demonstrated by the bilateral generalized reduction in mfERG N1 and P1 response amplitudes. Behbehani et al. [60] also performed mfERG in patients with ethambutolassociated visual loss in order to investigate whether there is outer retinal toxicity due to ethambutol therapy. A significantly reduced P1 amplitude was found in ethambutol-treated patients, indicating ethambutol-induced retinal dysfunction at the level of photoreceptors and bipolar cells, and it was not restricted to the optic nerve as so far considered. Similarly, Kardon et al. [61] found decreased mfERG amplitudes mainly in the central part of the VF in patients with ethambutol toxicity. Subsequently, Liu et al. [62] demonstrated that mfERG abnormalities correspond to the bitemporal VF defects which developed in 2 patients under ethambutol. The authors suggested that the visual impairment may be entirely attributable to retinal rather than optic nerve toxicity. Lai et al. [63] studied mfERG findings of visually asymptomatic patients receiving ethambutol therapy and observed normal N1 and $\mathrm{P} 1$ response amplitudes in all ring eccentricities but significant delays in P1 implicit times of the more peripheral rings $4-5$. The findings indicated mild abnormalities in the peripheral macula of patients on ethambutol therapy when no visual symptoms occur. No correlation was found between the mfERG changes and the dose or duration of ethambutol therapy. Kandel et al. [64] studied the visual effects of ethambutol in 88 eyes performing mfERG before and 2 months after the initiation of the drug. Reduced P1 amplitudes and increased P1 latencies were observed in all rings 2 months after therapy with ethambutol. mfERG changes seem to be reversible after ethambutol cessation $[59,61]$.

The above electrophysiological studies suggest that mfERG may be valuable for the diagnosis of subclinical cases of ethambutol toxicity and for monitoring patients under ethambutol therapy with ocular toxicity.

\section{Interferon-a Retinopathy}

The combination of antiviral drugs like interferon- $\alpha$ and ribavirin has been used in the treatment of chronic hepatitis C. Ischemic retinopathy manifested as cotton wool spots, and retinal hemorrhage is a well-recognized complication of interferon therapy $[65,66]$.

mfERG has been used to assess retinal dysfunction associated with interferon- $\alpha$ therapy. Chisholm et al. [67] studied mfERG recordings of 10 patients while on therapy with pegylated interferon- $\alpha$ for chronic hepatitis C. The latencies of the averaged mfERG responses showed abnormalities in one or both eyes in 5 of 10 patients. Delays were more common in the central than the peripheral retina. No correlation between the appearance of cotton wool spots and/or retinal hemorrhage and abnormalities on mfERG testing was found. Abnormal mfERG responses were observed in 4 asymptomatic patients with normal fundus appearance, suggesting that $\mathrm{mfERG}$ is a sensitive method of testing subclinical retinal toxicity during antiviral therapy with interferon- $\alpha$. 


\section{Tamoxifen Retinopathy}

Tamoxifen is an oral antiestrogen most commonly used at low dosages ( $20 \mathrm{mg}$ daily) in the adjuvant treatment of breast cancer. High-dose tamoxifen chemotherapy may also be applied for the treatment of brain malignancies. Several ocular findings such as corneal opacities, cataract, cystoid macular edema, macular hole, optic neuritis and retinopathy have been described as adverse effects of tamoxifen. Retinopathy is characterized by small, white, refractile opacities within the retina [68].

Salomao et al. [69] used mfERG to study retinal toxicity in 30 visually asymptomatic patients taking low-dosage tamoxifen and found that the central retinal function was not affected even after 2 years of tamoxifen use or in cases with refractile retinal crystals. The researchers suggested that mfERG in not a sufficiently sensitive method for detecting tamoxifen-associated changes. On the contrary, Ritter et al. [70] reported on 8 patients taking lowdosage tamoxifen, including 7 patients complaining of visual disturbances and 1 patient presenting with crystalline deposits in the cornea and macula, and found mfERG changes in 5 patients. The authors recommended electrophysiological examination in symptomatic patients on tamoxifen. Similarly, Srikantia et al. [71] performed mfERG in a patient with bilateral crystalline maculopathy associated with tamoxifen use and showed reduced responses in paracentral and pericental areas of both eyes.

Because of the variability in $\mathrm{mfERG}$ findings of the cases of tamoxifen retinopathy reported, further studies are needed to establish the degree of retinal toxicity associated with tamoxifen use.

\section{Digoxin Retinopathy}

Digoxin is a cardiac glucoside commonly used for the treatment of congestive cardiac failure. Visual disturbances are the most common symptoms of digitalis toxicity and often the initial signs [72-74]. A common ocular complaint is xanthopsia, but blurring, central scotomas, photopsia and altered color perception have also been reported $[72,73,75]$.

Recently, Renard et al. [76] reported a diffuse decrease in amplitude on mfERG mostly in the central $10^{\circ}$ of VF in a digoxin-treated patient complaining of blurry vision, dyschromatopsia and visual hallucinations. After cessation of the drug, visual symptoms progressively improved but mfERG changes persisted.

\section{Sildenafil Retinopathy}

Sildenafil is an oral medication used for the treatment of male erectile dysfunction by the inhibition of phosphodiesterase (PDE) 5 in the corpus cavernosum and the subsequent facilitation of penile erection. In vitro studies have shown that PDE6, found predominantly in retinal photoreceptors, is a key enzyme in the phototransduction cascade which is also inhibited by sildenafil. The inhibition efficacy is about $1 / 10$ of that for PDE5. The approved doses of sildenafil for the as-needed treatment of erectile dysfunction are 25, 50 and $100 \mathrm{mg}$ [77]. Various ocular side effects have been reported, such as some unusual visual disturbances, including a sense of bluish vision, increased perception of brightness, blurred vision and photophobia [78, 79].

Human ERG studies have shown mild responses in healthy individuals after a single, large dose of sildenafil. Full-field ERG and mfERG were used to evaluate the acute effects of a single dose of 100 or $200 \mathrm{mg}$ sildenafil on retinal function of healthy subjects. The full-field ERG and mfERG responses revealed transiently increased photopic but not scotopic implicit times, and delayed and attenuated waveforms across the posterior pole, respectively, suggesting a depression of cone function both at macula and periphery level [80]. Another study used ERG and mfERG recording to assess the visual effects of a chronic, daily, high dose $(100 \mathrm{mg})$ of sildenafil and revealed only a modest increase in cone implicit time [81]. However, mfERG demonstrated a small increase in amplitude and decrease in implicit time $12 \mathrm{~h}$ after the last sildenafil dose, which returned $1 \mathrm{~h}$ after redosing, indicating a possible reversibility of the retinal abnormalities in the short term.

The mfERG findings revealed objective evidence that sildenafil may cause an acute, reversible macular dysfunction responsible for the transient visual disturbances described by the patients.

\section{Canthaxanthin Retinopathy}

Canthaxanthin is a vitamin A or carotenoid derivative that can be used as an oral tanning agent. Patients typically are asymptomatic, but cases of multiple, gold-colored, glistening, intraretinal crystalline deposits surrounding the macula and papilla have been reported. The crystals are typically distributed in a donut configuration surrounding the fovea and appear after high-dose ingestion of canthaxanthin [82]. 
Hueber et al. [83] described the long-term outcome of canthaxanthin retinopathy in 7 asymptomatic patients with small golden particles near the macula using perimetry, OCT, FAF and fluorescein angiography in all patients and additional EOG, ERG and mfERG in 3 patients. EOG was normal in all 3 patients. Scotopic ERG and mfERG were normal in 2 patients and subnormal, but not statistically significant, when compared with age-matched controls in 1 patient. Photopic and $30-\mathrm{Hz}$ flicker ERG revealed subnormal but nonsignificant amplitudes compared to controls in 2 patients and normal response in 1 patient. Perimetry, OCT, FAF and fluorescein angiography were normal in all patients, and complete disappearance of the golden particles took approximately 20 years. However, further studies with larger samples are needed to confirm the role of mfERG in the diagnosis of canthaxanthin retinopathy.

\section{Amiodarone Retinopathy}

Amiodarone is an antiarrhythmic drug indicated for cardiac arrhythmias and congestive heart failure, and in patients after acute myocardial infarction. The ocular side effects of amiodarone involve mainly the anterior segment, including characteristic whorled corneal epithelial opacities [84].

Based on the observation that several patients receiving amiodarone developed atypical pigmentary maculopathy, Shaikh et al. [85] evaluated the mfERG responses of 11 patients on chronic amiodarone therapy in order to investigate whether there are any correlations between this drug and the retinal disease. mfERG P1 amplitudes were normal in all but 2 patients. One patient developed a subnormal value in the central fovea and the other revealed decreased amplitudes at all eccentricities across the posterior pole. $\mathrm{P} 1$ wave latencies were mildly prolonged in the foveal or parafoveal region in 3 patients, and across the posterior pole in 2 patients. The authors concluded that the mildly abnormal findings in a few patients showed no consistent pattern to suggest a cause of toxicity and could not be correlated with amiodarone exposure.

\section{Nefazodone Retinopathy}

Nefazodone is an antidepressant drug that blocks postsynaptic type- 2 serotonin (5HT2) receptors. It has been associated with blurred vision, VF defects, photophobia, diplopia and other visual disturbances [86].
In order to investigate the nature and site of the neural deficits in a patient with visual disturbances associated with nefazodone, Luu et al. [86] performed both mfERG and multifocal VEP (mfVEP) recordings. The visual side effects included reduced visual acuities, reduced color vision and VF constriction in both eyes. MfERG revealed a severe depression in macular responses in both eyes, and mfVEP showed central depression in one eye. The authors reported that the losses indicated by the mfERG were more severe than those of mfVEP, suggesting that the primary locus of neural damage was retinal, which was likely to have resulted from neurotoxicity. The use of full-field ERG was inadequate for identifying the current lesions in this case.

\section{Conclusion}

mfERG is widely used for the evaluation of drug-induced retinopathy. It is particularly useful for the diagnosis of retinal toxicity limited to the central retina, as fullfield ERG is usually normal in these cases, and the objective evaluation of disease severity. In some cases, such as in CQ, HCQ, VGB, interferon-a, ethambutol and sildenafil toxicity, retinal dysfunction may occur without structural abnormalities, and mfERG can provide an objective functional assessment of retinal toxicity. It is also able to detect the location and extent of retinal dysfunction in such cases. Moreover, the mfERG examination is helpful for the differential diagnosis or the confirmation of diagnosis of toxicity. It is valuable in monitoring disease progression or recovery after drug cessation. mfERG findings have demonstrated that early retinal toxicity is reversible in HCQ, ethambutol and sildenafil retinopathy, and may persist in deferoxamine retinopathy after drug cessation. However, further electrophysiological studies are needed in order to establish its role in the assessment of retinal function in patients treated with tamoxifen, canthaxanthin, digoxin and nefazodone.

\section{Disclosure Statement}

The authors have no conflicts of interest to disclose. 


\section{References}

$>1$ Sutter EE, Tran D: The field topography of 14 Dolan FM, Parks S, Hammer H, Keating D: ERG components in man - I. The photopic luminance response. Vision Res 1992;32:433446.

$>2$ Hood DC, Bach M, Brigell M, Keating D, Kondo M, Lyons JS, Marmor MF, McCulloch DL, Palmowski-Wolfe AM: ISCEV standard for clinical multifocal electroretinography (mfERG) (2011 edition). Doc Ophthalmol 2012;124:1-13.

3 McCulloch DL, Marmor MF, Brigell MG, Hamilton R, Holder GE, Tzekov R, Bach M: ISCEV standard for full-field clinical electroretinography (2015 update). Doc Ophthalmol 2015;130:1-12.

4 Lai TY, Chan WM, Lai RY, Ngai JW, Li H, Lam DS: The clinical applications of multifocal electroretinography: a systematic review. Surv Ophthalmol 2007;52:61-96.

5 Young B, Eggenberger E, Kaufman D: Current electrophysiology in ophthalmology: a review. Curr Opin Ophthalmol 2012;23:497505.

6 Kellner U, Renner AB, Tillack H: Fundus autofluorescence and mfERG for early detection of retinal alterations in patients using chloroquine/hydroxychloroquine. Invest Ophthalmol Vis Sci 2006;47:3531-3538.

$>7$ Missner S, Kellner U: Comparison of different screening methods for chloroquine/hydroxychloroquine retinopathy: multifocal electroretinography, color vision, perimetry, ophthalmoscopy, and fluorescein angiography. Graefes Arch Clin Exp Ophthalmol 2012;250 319-325.

-8 Gupta S, Midha N, Gogia V, Sahay P, Pandey V, Venkatesh P: Sensitivity of multifocal electroretinography (mfERG) in detecting siderosis. Can J Ophthalmol 2015;50:485-490.

$\checkmark$ Moschos MM, Gonidakis F, Varsou E, Markopoulos I, Rouvas A, Ladas I, Papadimitriou GN: Anatomical and functional impairment of the retina and optic nerve in patients with anorexia nervosa without vision loss. Br J Ophthalmol 2011;95:1128-1133.

$>10$ Moschos MM, Triglianos A, Rotsos T, Papadimitriou S, Margetis I, Minogiannis $\mathrm{P}$, Moschos M: Tilted disc syndrome: an OCT and mfERG study. Doc Ophthalmol 2009; 119:23-28.

-11 Moschos MM, Chatziralli IP, Koutsandrea C, Siasou G, Droutsas D: Assessment of the macula in keratoconus: an optical coherence tomography and multifocal electroretinography study. Ophthalmologica 2013;229:203207.

-12 Kretschmann U, Bock M, Gockeln R, Zrenner E: Clinical applications of multifocal electroretinography. Doc Ophthalmol 2000;100:99_ 113.

$>13$ Holder GE: Electrophysiological assessment of optic nerve disease. Eye (Lond) 2004;18: 1133-1143. The wide field multifocal electroretinogram reveals retinal dysfunction in early retinitis pigmentosa. Br J Ophthalmol 2002;86:480 481.

15 Dolan FM, Parks S, Keating D, Dutton GN: Wide field multifocal and standard full field electroretinographic features of hemi retinal vein occlusion. Doc Ophthalmol 2006;112: 43-52.

16 Dolan FM, Gavin M, Parks S, Keating D: Recovery of visual function in a patient with birdshot chorioretinitis. $\mathrm{Br} \mathrm{J}$ Ophthalmol 2003;87:657-658

17 Gonzalez P, Sills GJ, Parks S, Kelly K, Stephen LJ, Keating D, Dutton GN, Brodie MJ: Binasal visual field defects are not specific to vigabatrin. Epilepsy Behav 2009;16:521-526.

18 McDonagh J, Stephen LJ, Dolan FM, Parks S, Dutton GN, Kelly K, Keating D, Sills GJ, Brodie MJ: Peripheral retinal dysfunction in patients taking vigabatrin. Neurology 2003;61: 1690-1694.

19 Santaella RM, Fraunfelder FW: Ocular adverse effects associated with systemic medications: recognition and management. Drugs 2007;67:75-93.

20 Tzekov R: Ocular toxicity due to chloroquine and hydroxychloroquine: electrophysiological and visual function correlates. Doc Ophthalmol 2005;110:111-120.

21 Raines MF, Bhargava SK, Rosen ES: The blood-retinal barrier in chloroquine retinopathy. Invest Ophthalmol Vis Sci 1989;30: 1726-1731.

22 Maturi RK, Folk JC, Nichols B, Oetting TT, Kardon RH: Hydroxychloroquine retinopathy. Arch Ophthalmol 1999;117:1262-1263.

23 Michaelides M, Stover NB, Francis PJ, Weleber RG: Retinal toxicity associated with hydroxychloroquine and chloroquine: risk factors, screening, and progression despite cessation of therapy. Arch Ophthalmol 2011;129: 30-39.

24 Maturi RK, Yu M, Weleber RG: Multifocal electroretinographic evaluation of long-term hydroxychloroquine users. Arch Ophthalmol 2004;122:973-981.

25 Kellner U, Kraus H, Foerster MH: Multifocal ERG in chloroquine retinopathy: regional variance of retinal dysfunction. Graefes Arch Clin Exp Ophthalmol 2000;238:94-97.

26 So SC, Hedges TR, Schuman JS, Quireza ML: Evaluation of hydroxychloroquine retinopathy with multifocal electroretinography. Ophthalmic Surg Lasers Imaging 2003;34:251-258.

27 Lai TY, Chan WM, Li H, Lai RY, Lam DS: Multifocal electroretinographic changes in patients receiving hydroxychloroquine therapy. Am J Ophthalmol 2005;140:794-807.

28 Chang WH, Katz BJ, Warner JE, Vitale AT, Creel D, Digre KB: A novel method for screening the multifocal electroretonogram in patients using hydroxychloroquine. Retina 2008;28:1478-1486.
29 Lyons JS, Severns ML: Using multifocal ERG ring ratios to detect and follow Plaquenil retinal toxicity: a review: Review of mfERG ring ratios in Plaquenil toxicity. Doc Ophthalmol 2009;118:29-36.

30 Tsang AC, Ahmadi PS, Virgili G, Gottlieb CC, Hamilton J, Coupland SG: Hydroxychloroquine and chloroquine retinopathy: a systematic review evaluating the multifocal electroretinogram as a screening test. Ophthalmology 2015;122:1239-1251.

31 Moschos MN, Moschos MM, Apostolopoulos M, Mallias JA, Bouros C, Theodossiadis GP: Assessing hydroxychloroquine toxicity by the multifocal ERG. Doc Ophthalmol 2004;108: 47-53.

32 Missner S, Kellner U: Comparison of different screening methods for chloroquine/hydroxychloroquine retinopathy: multifocal electroretinography, color vision, perimetry, ophthalmoscopy, and fluorescein angiography. Graefes Arch Clin Exp Ophthalmol 2012;250: 319-325.

33 Greenstein VC, Amaro-Quireza L, Abraham ES, Ramachandran R, Tsang SH, Hood DC: A comparison of structural and functional changes in patients screened for hydroxychloroquine retinopathy. Doc Ophthalmol 2015; 130:13-23.

34 Marmor MF, Kellner U, Lai TY, Lyons JS, Mieler WF: Revised recommendations on screening for chloroquine and hydroxychloroquine retinopathy. Ophthalmology 2011; 118:415-422.

35 Browning DJ, Lee C: Relative sensitivity and specificity of 10-2 visual fields, multifocal electroretinography, and spectral domain optical coherence tomography in detecting hydroxychloroquine and chloroquine retinopathy. Clin Ophthalmol 2014;8:1389-1399.

36 Leung LS, Neal JW, Wakelee HA, Sequist LV, Marmor MF: Rapid onset of retinal toxicity from high-dose hydroxychloroquine given for cancer therapy. Am J Ophthalmol 2015; 160:799-805.

37 Penrose PJ, Tzekov RT, Sutter EE, Fu AD, Allen AW Jr, Fung WE, Oxford KW: Multifocal electroretinography evaluation for early detection of retinal dysfunction in patients taking hydroxychloroquine. Retina 2003;23: 503-512.

38 Leung LS, Neal JW, Wakelee HA, Sequist LV, Marmor MF: Rapid onset of retinal toxicity from high-dose hydroxychloroquine given for cancer therapy. Am J Ophthalmol 2015; 160:799-805.

39 Moschos MM, Nitoda E, Chatziralli IP, Gatzioufas Z, Koutsandrea C, Kitsos G: Assessment of hydroxychloroquine maculopathy after cessation of treatment: an optical coherence tomography and multifocal electroretinography study. Drug Des Devel Ther 2015;9: 2993-2999. 
40 Malmgren K, Ben Menachem E, Frisen L: Vi- 55 Bansal V, Elgarbly I, Ghanchi FD, Atkinson gabatrin visual toxicity: evolution and dose dependence. Epilepsia 2001;42:609-615.

-41 Maguire MJ, Hemming K, Wild JM, Hutton JL, Marson AG: Prevalence of visual field loss following exposure to vigabatrin therapy: a systematic review. Epilepsia 2010;51:24232431.

\$2 Lawden MC, Eke T, Degg C, Harding GF, Wild JM: Visual field defects associated with vigabatrin therapy. J Neurol Neurosurg Psychiatry 1999;67:716-722.

43 Harding GF, Wild JM, Robertson KA, Lawden MC, Betts TA, Barber C, Barnes PM: Electro-oculography, electroretinography, visual evoked potentials, and multifocal electroretinography in patients with vigabatrin-attributed visual field constriction. Epilepsia 2000; 41:1420-1431.

-44 Coupland SG, Zackon DH, Leonard BC, Ross TM: Vigabatrin effect on inner retinal function. Ophthalmology 2001;108:1493-1496.

-45 Ponjavic V, Andreasson S: Multifocal ERG and full-field ERG in patients on long-term vigabatrin medication. Doc Ophthalmol 2001;102:63-72.

-46 Daneshvar H, Racette L, Coupland SG, Kertes PJ, Guberman A, Zackon D: Symptomatic and asymptomatic visual loss in patients taking vigabatrin. Ophthalmology 1999;106: 1792-1798.

-47 Johnson MA, Krauss GL, Miller NR, Medura M, Paul SR: Visual function loss from vigabatrin: effect of stopping the drug. Neurology 2000;55:40-45.

-48 Besch D, Kurtenbach A, Apfelstedt-Sylla E, Sadowski B, Dennig D, Asenbauer C, Zrenner E, Schiefer U: Visual field constriction and electrophysiological changes associated with vigabatrin. Doc Ophthalmol 2002;104:151170 .

49 Brittenham GM: Iron-chelating therapy for transfusional iron overload. N Engl J Med 2011;364:146-156.

50 Di Nicola M, Barteselli G, Dell'Arti L, Ratiglia R, Viola F: Functional and structural abnormalities in deferoxamine retinopathy: a review of the literature. Biomed Res Int 2015; 2015:249617

51 Porter JB, Huehns ER: The toxic effects of desferrioxamine. Baillieres Clin Haematol 1989; 2:459-474.

52 Lakhanpal V, Schocket SS, Jiji R: Deferoxamine (Desferal)-induced toxic retinal pigmentary degeneration and presumed optic neuropathy. Ophthalmology 1984;91:443451.

53 Haimovici R, D’Amico DJ, Gragoudas ES, Sokol S: The expanded clinical spectrum of deferoxamine retinopathy. Ophthalmology 2002;109:164-171.

54 Viola F, Barteselli G, Dell'Arti L, Vezzola D, Villani E, Mapelli C, Zanaboni L, Cappellini $\mathrm{MD}$, Ratiglia R: Abnormal fundus autofluorescence results of patients in long-term treatment with deferoxamine. Ophthalmology 2012;119:1693-1700 amine. Eur J Haematol 2003;70:420-421.

56 Schmidt D, Finke J: Bull's-eye maculopathy with deferoxamine treatment (in German). Klin Monbl Augenheilkd 2004;221:204-209.

57 Kertes PJ, Lee TK, Coupland SG: The utility of multifocal electroretinography in monitoring drug toxicity: deferoxamine retinopathy. Can J Ophthalmol 2004;39:656-661.

58 Fraunfelder FW, Sadun AA, Wood T: Update on ethambutol optic neuropathy. Expert Opin Drug Saf 2006;5:615-618.

59 Lai TY, Chan WM, Lam DS, Lim E: Multifocal electroretinogram demonstrated macular toxicity associated with ethambutol related optic neuropathy. Br J Ophthalmol 2005;89: 774-775.

60 Behbehani RS, Affel EL, Sergott RC, Savino PJ: Multifocal ERG in ethambutol associated visual loss. Br J Ophthalmol 2005;89:976-982.

61 Kardon RH, Morrisey MC, Lee AG: Abnormal multifocal electroretinogram (mfERG) in ethambutol toxicity. Semin Ophthalmol 2006;21:215-222.

62 Liu Y, Dinkin MJ, Loewenstein JI, Rizzo JF III, Cestari DM: Multifocal electroretinographic abnormalities in ethambutol-induced visual loss. J Neuroophthalmol 2008;28:278-282.

63 Lai TY, Ngai JW, Lai RY, Lam DS: Multifocal electroretinography changes in patients on ethambutol therapy. Eye (Lond) 2009;23: 1707-1713.

64 Kandel H, Adhikari P, Shrestha GS, Ruokonen $\mathrm{EL}$, Shah DN: Visual function in patients on ethambutol therapy for tuberculosis. J Ocul Pharmacol Ther 2012;28:174-178.

65 Guyer DR, Tiedeman J, Yannuzzi LA, Slakter JS, Parke D, Kelley J, Tang RA, Marmor M, Abrams G, Miller JW, Gragoudas ES: Interferon-associated retinopathy. Arch Ophthalmol 1993;111:350-356.

66 Jain K, Lam WC, Waheeb S, Thai Q, Heathcote J: Retinopathy in chronic hepatitis $\mathrm{C}$ patients during interferon treatment with ribavirin. Br J Ophthalmol 2001;85:1171-1173. Keating D, Gavin M, Mills PR: Retinal toxicity during pegylated alpha-interferon therapy for chronic hepatitis C: a multifocal electroretinogram investigation. Aliment Pharmacol Ther 2005;21:723-732.

68 Watanabe SE, Berezovsky A, Motono M, Sacai PY, Pereira JM, Sallum JM, Gebrim LH, Salomao SR: Retinal function in patients treated with tamoxifen. Doc Ophthalmol 2010;120:137-143.

69 Salomao SR, Watanabe SE, Berezovsky A, Motono M: Multifocal electroretinography, color discrimination and ocular toxicity in tamoxifen use. Curr Eye Res 2007;32:345352. PL: Bull's eye maculopathy with deferox-

67 Chisholm JA, Williams G, Spence E, Parks S
70 Ritter C, Renner AB, Wachtlin J, Bechrakis NE, Krause L: Tamoxifen retinopathy: a case series of clinical and functional data (in German). Ophthalmologe 2008;105:544-549.

71 Srikantia N, Mukesh S, Krishnaswamy M: Crystalline maculopathy: a rare complication of tamoxifen therapy. J Cancer Res Ther 2010; 6:313-315.

72 Lely AH, van Enter CH: Large-scale digitoxin intoxication. Br Med J 1970;3:737-740.

73 Butler VP Jr, Odel JG, Rath E, Wolin MJ, Behrens MM, Martin TJ, Kardon RH, Gouras P: Digitalis-induced visual disturbances with therapeutic serum digitalis concentrations. Ann Intern Med 1995;123:676-680.

74 Robertson DM, Hollenhorst RW, Callahan JA: Ocular manifestations of digitalis toxicity. Discussion and report of three cases of central scotomas. Arch Ophthalmol 1966;76:640645 .

75 Lawrenson JG, Kelly C, Lawrenson AL, Birch $\mathrm{J}$ : Acquired colour vision deficiency in patients receiving digoxin maintenance therapy. Br J Ophthalmol 2002;86:1259-1261.

76 Renard D, Rubli E, Voide N, Borruat FX Rothuizen LE: Spectrum of digoxin-induced ocular toxicity: a case report and literature review. BMC Res Notes 2015;8:368.

$\checkmark 77$ Cordell WH, Maturi RK, Costigan TM, Marmor MF, Weleber RG, Coupland SG, Danis RP, McGettigan JW Jr, Antoszyk AN, Klise S, Sides GD: Retinal effects of 6 months of daily use of tadalafil or sildenafil. Arch Ophthalmol 2009; 127:367-373.

78 Marmor MF, Kessler R: Sildenafil (viagra) and ophthalmology. Surv Ophthalmol 1999; 44:153-162.

79 Fraunfelder FW: Visual side effects associated with erectile dysfunction agents. Am J Ophthalmol 2005; 140:723-724.

80 Luu JK, Chappelow AV, McCulley TJ, Marmor MF: Acute effects of sildenafil on the electroretinogram and multifocal electroretinogram. Am J Ophthalmol 2001;132:388394

-81 Zoumalan CI, Zamanian RT, Doyle RL, Marmor MF: ERG evaluation of daily, high-dose sildenafil usage. Doc Ophthalmol 2009;118: 225-231.

82 Drenser K, Sarraf D, Jain A, Small KW: Crystalline retinopathies. Surv Ophthalmol 2006; 51:535-549.

83 Hueber A, Rosentreter A, Severin M: Canthaxanthin retinopathy: long-term observations. Ophthalmic Res 2011;46:103-106.

84 Orlando RG, Dangel ME, Schaal SF: Clinical experience and grading of amiodarone keratopathy. Ophthalmology 1984;91:1184-1187.

85 Shaikh S, Shaikh N, Chun SH, Spin JM, Blumenkranz MS, Marmor MF: Retinal evaluation of patients on chronic amiodarone therapy. Retina 2003;23:354-359.

86 Luu C, Kiely P, Crewther D, Kowal L, Crewther S: Central and peripheral vision loss associated with nefazodone usage. Doc Ophthalmol 2003;106:319-325.
mfERG to Assess Drug-Induced Retinopathy
Ophthalmic Res 2016;56:169-177 